\title{
Minimum Cross Entropy Method with the Constraints of Uncertain Autocorrelations
}

\author{
K. Nakamuro, K. Tanaka, Y. Togawa \\ and S. Sugimoto \\ Department of Electrical and Electronic Engineering, Ritsumeikan University \\ Noji-Higashi, Kusatsu City, Shiga 525-8577 Japan \\ Tel: +81-(0)77-561-2673, FAX: +81-(0)77-561-2663 \\ e-mail: sugimoto@se.ritsumei.ac.jp
}

\begin{abstract}
In this paper, we present a minimum cross entropy spectrum estimation method with the constraints of uncertain autocorrelations for the continuous spectrum (C-MCEMU) [1]. Then, we derive consistency of the proposed spectral method and a minimum cross entropy method for the continuous spectrum with the constraints of known autocorrelations (C-MCEM). That is, when $\sigma^{2}=0$ in C-MCEMU, we derive that the proposed spectral estimation method corresponds to C-MCEM.
\end{abstract}

\section{Introduction}

A minimum cross entropy spectrum estimation method (MCEM) has been proposed by Shore and Johnson $[2,3]$, that is an extension of the maximum entropy spectral estimation method (MEM) by Burg [3, 4]. The original MCEM has been extended to more practical and general directions in $[5,6]$. In particular, C-MCEM has been proposed, based on Shore and Johnson's MCE method. Subsequently, we presented the MCEM with the constraints of uncertain autocorrelations [1] and derive the computational algorithms for C-MCEM with the constraints of uncertain autocorrelations.

In Section 2, we reference to C-MCEM with the constraints of known autocorrelations and one with the constraints of uncertain autocorrelations. Then, we derive consistency of them. In Section 3, we compare and consider the estimated results of proposed method, C-MCEM, Burg method.

\section{Spectrum Estimation by MCEM}

Let $\left\{\tilde{x}_{t}\right\}$ and $\left\{x_{t}\right\}(t=1,2, \ldots, L)$ be independent stationary Gaussian time series with their joint probability density functions $p\left(\underline{\tilde{x}}_{I}\right)$ and $q\left(\underline{x}_{T}\right)$, respectively, where $\underline{\tilde{x}}_{T}$, and $\underline{x}_{I}$ are used in place of $\left\{\tilde{x}_{1}, \tilde{x}_{2}, \ldots, \tilde{x}_{\tau}\right\}$ and $\left\{x_{1}, x_{2}, \ldots, x_{\tau}\right\}$, respectively. Then the cross entropy rate between two processes is defined by

$$
\mathcal{H}\left(\underline{x}_{t}, \underline{\tilde{x}}_{t}\right)=\lim _{L \rightarrow \infty} \frac{1}{L} \mathcal{H}_{I}\left(\underline{x}_{L}, \underline{\tilde{x}}_{L}\right)
$$

where $\mathcal{H}_{I}\left(\underline{x}_{L}, \underline{\tilde{x}}_{L}\right)$ is the cross entropy between two probability density functions $p\left(\underline{x}_{L}\right)$ and $q\left(\underline{x}_{\tau}\right)$ defined as

$$
\mathcal{H}_{I}\left(\underline{x}_{L}, \underline{\tilde{x}}_{L^{\prime}}\right)=\int q\left(\underline{x}_{L}\right) \ln \frac{q\left(\underline{x}_{\tau}\right)}{p\left(\underline{x}_{L}\right)} d \underline{x}_{L}
$$

and it is also called the Kullback-Leibler information measure of discrimination. Assuming zero means, its cross entropy rate between two independent stationary Gaussian time series $\left\{\tilde{x}_{t}\right\}$ and $\left\{x_{t}\right\}$ has been shown that

$$
\mathcal{H}\left(\underline{x}_{t}, \underline{\tilde{x}}_{t}\right)=\frac{1}{2 \pi} \int_{-\pi}^{\pi}\left\{-\ln \frac{S_{q}(\omega)}{S_{p}(\omega)}+\frac{S_{q}(\omega)}{S_{p}(\omega)}-1\right\} d \omega(3)
$$

where $S_{p}(\omega)$ and $S_{q}(\omega)$ are power spectrum density functions of $\left\{\tilde{x}_{t}\right\}$ and $\left\{x_{t}\right\}$, respectively $[5,7]$. 


\subsection{C-MCEM}

We assume that the prior spectrum $S_{p}(\omega)$ generated from $N$ th order AR models and $M+1$ autocorrelations $\left\{R_{0}, \ldots, R_{M}\right\}$ of $\left\{x_{t}\right\}$ satisfying the following equation.

$$
R_{k}=\frac{1}{2 \pi} \int_{-\pi}^{\pi} S_{q}(\omega) e^{j \omega k} d \omega,|k|=0, \ldots, M .
$$

By principle of $\mathrm{MCE}$, the posterior power spectrum $S_{q}(\omega)$ is determined by minimizing the cross entropy rate (3) under the constrain (4). This problem can be solved by the Lagrange multipliers technique and we obtain the estimated spectrum $\hat{S}_{q}(\omega)$ as

$$
\hat{S}_{q}(\omega)=\frac{S_{p}(\omega)}{1+S_{p}(\omega) \sum_{k=-M}^{M} \lambda_{k} e^{j \omega k}}
$$

where $\left\{\lambda_{k}\right\}$ is Lagrange multipliers [5]. Let us assume that the prior spectrum $S_{p}(\omega)$ and the posterior spectrum $S_{q}(\omega)$ are given as an all-pole type rational function. That is, they generated from $N$ th order prior AR models and $\max (M, N)$ th order posterior $\mathrm{AR}$ models, such as

$\left.S_{p}(\omega) \equiv \frac{\sigma_{p}^{2}}{A\left(z^{-1}\right) A^{*}\left(z^{-1}\right)}\right|_{z=e^{j \omega}}, A\left(z^{-1}\right)=\sum_{k=0}^{N} a_{k} z^{-k}(6)$
$\left.\hat{S}_{q}(\omega) \equiv \frac{\sigma_{q}^{2}}{B\left(z^{-1}\right) B^{*}\left(z^{-1}\right)}\right|_{z=e^{j \omega}}, B\left(z^{-1}\right)=\sum_{k=0}^{\max (M, N)} b_{k} z^{-k}(7)$

where $\left\{a_{k}\right\},\left\{b_{k}\right\}, \sigma_{p}^{2}$ and $\sigma_{q}^{2}$ are real constants, and $a_{0}=1, b_{0}=1$. Here we consider about two cases, one is $M \geq N$. In this case, the posterior AR model's order is $M$ and $M+1$ unknown parameters $\left\{b_{1}, \ldots, b_{M}\right.$, $\left.\hat{\sigma}^{2}\right\}$ exist. These $M+1$ parameters can be determined from $M+1$ constraints (4) and by solving the Yule-Walker equation. Another case is $M<N$. In this case, the posterior AR model's order is $N$. From (5), (6) and (7), it can be shown that the following relations hold.

$$
\begin{aligned}
\frac{\sigma_{q}^{2}}{\sigma_{p}^{2}}\left(\sum_{k=r}^{N} a_{k} a_{k-r}+\sigma_{p}^{2} \lambda_{r}\right) & =\sum_{\substack{k=r \\
r}}^{N} b_{k} b_{k-r}, \ldots, M .
\end{aligned}
$$

$\frac{\sigma_{q}^{2}}{\sigma_{p}^{2}} \sum_{k=r}^{N} a_{k} a_{k-r}=\sum_{k=r}^{N} b_{k} b_{k-r}, r=M+1, \ldots, N$

Lagrange multipliers $\left\{\lambda_{k}\right\}$ are shown as follows [5].

$$
\begin{array}{r}
\lambda_{r}=\frac{1}{\sigma_{q}^{2}}\left(\sum_{k=r}^{N} b_{k} b_{k-r}\right)-\frac{1}{\sigma_{p}^{2}}\left(\sum_{k=r}^{N} a_{k} a_{k-r}\right) \\
r=0, \ldots, M(10)
\end{array}
$$

Furthermore, we hold the Yule-Walker equation as follows.

$$
\sum_{k=0}^{N} R_{r-k} b_{k}=\sigma_{q}^{2} \delta_{r}, \quad r=0, \ldots, N
$$

where $\delta_{r}$ denotes Kronecker's delta function. Therefore, we can obtain estimated posterior spectrum $\hat{S}_{q}(\omega)$ by solving totally $2 N-M+1$ th simultaneous equations in (9) and (11), which contain unknown parameters, $\left\{b_{1}, \ldots, b_{N}, R_{M+1}, \ldots, R_{N}, \sigma_{q}^{2}\right\}$, while the parameters $\left\{a_{1}, \ldots, a_{N}, \sigma_{p}^{2}\right\}$ are known from the prior information and $\left\{R_{0}, \ldots, R_{M}\right\}$ are also known from the constrains. In order to show a concrete numerical computation method, the following vector functions are defined to the equation of these equations.

$$
\begin{aligned}
\underline{f}(\underline{y}) & \equiv\left[f_{1}(\underline{y}), \ldots, f_{2 N-M+1}(\underline{y})\right]^{T} \\
\underline{y} & \equiv\left[b_{1}, \ldots, b_{N}, R_{M+1}, \ldots, R_{N}, \sigma_{q}^{2}\right]^{T} \\
f_{r}(\underline{y}) & =-\sum_{k=0}^{N} R_{r-k-1} b_{k}+\sigma_{q}^{2} \delta_{r-1} \\
f_{r}(\underline{y}) & =\sum_{k=0}^{2 N-M+1-r} b_{k} b_{k+r+M-N-1} \\
& \quad-\frac{\sigma_{q}^{2}}{\sigma_{p}^{2}} \sum_{k=0}^{2 N-M+1-r} a_{k} a_{k+r+M-N-1} \\
r & =N+2, \ldots, 2 N-M+1(15)
\end{aligned}
$$

Then the numerical procedure of solving the above problem is reduced to obtain the solution of

$$
\underline{f}(\underline{y})=\underline{0}
$$

and we use the Newton's method to solve this procedure. 


\subsection{C-MCEM with the constraints} of uncertain autocorrelations (C-MCEMU)

In the same way, we assume that a prior spectrum $S_{p}(\omega)$ generated from $N$ th order AR models and $M+1$ autocorrelations $\left\{R_{k}\right\}(k=0,1, \ldots, M)$ of $\left\{x_{t}\right\}$ satisfying the following equation,

$$
\sum_{k=-M}^{M} w_{k}\left(\frac{1}{2 \pi} \int_{-\pi}^{\pi} S_{q}(\omega) e^{j \omega k} d \omega-R_{|| k \mid}\right)^{2}=\sigma^{2}(17)
$$

where $w_{k}$ are positive weighted factors which represent accuracy of measurement of autocorrelations $R_{k}$ and $\sigma^{2}$ represents the total uncertainty of correlations. Also, the posterior power spectrum $S_{q}(\omega)$ is determined by minimizing the cross entropy rate (3) under the constraints (17). This problem can be solved by the Lagrange multipliers technique and we obtain the estimated spectrum $\hat{S}_{q}(\omega)$ as

$$
\hat{S}_{q}(\omega)=\frac{S_{p}(\omega)}{1+S_{p}(\omega) \sum_{k=-M}^{M} \beta_{k} e^{j \omega k}}
$$

where

$$
\beta_{k} \equiv 2 \alpha w_{k}\left(\frac{1}{2 \pi} \int_{-\pi}^{\pi} \hat{S}_{q}(\omega) e^{j \omega k} d \omega-R_{|k|}\right)
$$

and $\alpha$ is a Lagrange multiplier [5]. Furthermore like the case of known correlation function, let us assume that the prior spectrum $S_{p}(\omega)$ and the posterior spectrum $S_{q}(\omega)$ are generated from $N$ th order prior AR models and $\max (M, N)$ th order posterior AR. models. Here we consider the case of $M<N$. From (17), (6) and (7), we have

$$
\begin{gathered}
\frac{\sigma_{q}^{2}}{\sigma_{p}^{2}}\left(\sigma \sum_{k=r}^{N} a_{k} a_{k-r}+\sigma_{p}^{2} \sigma \beta_{r}\right)=\sigma \sum_{k=r}^{N} b_{k} b_{k-r}, \\
r=0, \ldots, M .(20) \\
\frac{\sigma_{q}^{2}}{\sigma_{p}^{2}} \sum_{k=r}^{N} a_{k} a_{k-r}=\sum_{k=r}^{N} b_{k} b_{k-r}, \\
r=M+1, \ldots, N .(21)
\end{gathered}
$$

From (17) and (18), Lagrange multiplier $\alpha$ is shown as follows.

$$
\alpha= \pm \frac{1}{2 \sigma} \sqrt{\sum_{n=-M}^{M} \frac{\beta_{n}^{2}}{w_{n}}}
$$

The following equation is obtained by substituting (22) into (19).

$$
\sigma \beta_{k}=\frac{w_{k}}{2 \pi} \sqrt{\sum_{n=-M}^{M} \frac{\beta_{n}^{2}}{w_{n}}}\left(\int_{-\pi}^{\pi} \hat{S}_{q}(\omega) e^{j \omega k} d \omega-R_{|| k \mid}\right)(23)
$$

We can obtain estimated posterior spectrum $\hat{S}_{q}(\omega)$ by solving totally $N+M+2$ th simultaneous equations in (21), (21) and (23), which contain unknown parameters, $\left\{b_{1}, \ldots, b_{N}, \sigma_{q}^{2}, \beta_{0}, \ldots, \beta_{M}\right\}$. In order to show a concrete numerical computation method, the following vector functions are defined to the equation of these equations.

$$
\begin{aligned}
& \underline{f}(\underline{y}) \equiv {\left[f_{1}(\underline{y}), \ldots, f_{N+M+2}(\underline{y})\right]^{T} } \\
& \underline{y} \equiv {\left[b_{1}, \ldots, b_{N}, \sigma_{q}^{2}, \beta_{0}, \ldots, \beta_{M}\right]^{T} } \\
& f_{r}(\underline{y})=\sigma \sum_{k=0}^{N-r+1} b_{k+r-1} b_{k} \\
& \quad-\frac{\sigma_{q}^{2}}{\sigma_{p}^{2}}\left[\sigma \sum_{k=0}^{N-r+1} a_{k+r-1} a_{k}+\sigma_{p}^{2} \sigma \beta_{r-1}\right] \\
& r=1, \ldots, M+1 \quad(26) \\
& f_{r}(\underline{y})=\sum_{k=0}^{N-r+1} b_{k+r-1} b_{k}-\frac{\sigma_{q}^{2}}{\sigma_{p}^{2}} \sum_{k=0}^{N-r+1} a_{k+r-1} a_{k} \\
& r=M+2, \ldots, N+1 \quad(27) \\
& f_{r}(\underline{y})=W_{r}\left(\int_{-\pi}^{\pi} \hat{S}_{q}(\omega) e^{j \omega(r-N-2)} d \omega-R_{|r-N-2|}\right) \\
& \quad r=N \beta_{r-N-2} \\
& r=2, \ldots, N+M+2
\end{aligned}
$$

where

$$
W_{r}=\frac{w_{r-N-2}}{2 \pi} \sqrt{\sum_{n=-M}^{M} \frac{\beta_{n}^{2}}{w_{n}}}
$$

and $\hat{S}_{q}(\omega)$ in (28) is given by (7). Therefore nonlinear simultaneous equations (26), (27), (28) can be expressed as follows.

$$
\underline{f}(\underline{y})=\underline{0}
$$


Next, we explain a concrete numerical procedure. First, in (17) $\sigma^{2},\left\{w_{k}\right\}$ are defined as

$$
w_{k} \equiv \frac{1}{\operatorname{Var}\left\{\hat{R}_{k}\right\}}, \quad|k|=0,1, \ldots, M
$$

where

$$
\begin{array}{r}
\hat{R}_{k}=\frac{1}{L-|k|} \sum_{t=1}^{L-|k|} x_{t} x_{t+|k|}, \quad E\left\{\hat{R}_{k}\right\}=R_{k},(32) \\
\begin{array}{r}
\operatorname{Var}\left\{\hat{R}_{k}\right\}=\frac{1}{L-|k|} \sum_{m=-(L-|k|-1)}^{L-|k|-1}\left(1-\frac{|m|+|k|}{L-|k|}\right) \\
\times\left(R_{m}^{2}+R_{m+k} R_{m-k}\right) \quad(33)
\end{array}
\end{array}
$$

and $\sigma^{2}=2 M+1$. From a posterior process, estimation of $M+1$ autocorrelations $\left\{R_{0}, \ldots, R_{M}\right\}$ and prior AR model parameters $\left\{a_{1}, \ldots, a_{N}\right\}$ are prepared as prior information. These known parameters $\left\{\sigma^{2}, w_{0}, \ldots, w_{M}, R_{0}, \ldots, R_{M}, a_{1}, \ldots, a_{N}, \sigma_{p}^{2}\right\}$ is substituted to (30). Since this problem can be solved by Newton's method, $\left\{b_{1}, \ldots, b_{N}, \sigma_{q}^{2}, \beta_{0}, \ldots, \beta_{M}\right\}$ can be known and $\hat{S}_{q}(\omega)$ can be obtained from (7) or (18).

\subsection{Consistency of C-MCEM and C-MCEMU}

We consider the case that autocorrelations are known, that is $\sigma=0$ in (17) and derive that the case is consistent with known autocorrelations method. Then, unknown parameters are as follows.

Table 1: Unknown Parameters

\begin{tabular}{|c|c|c|}
\hline & Unknown parameters & Number \\
\hline$\sigma \neq 0$ & $\left\{b_{1}, \ldots, b_{N}, \sigma_{q}^{2}, \beta_{0}, \ldots, \beta_{M}\right\}$ & $N+M+2$ \\
\hline$\sigma=0$ & $\left\{b_{1}, \ldots, b_{N}, R_{M+1}, \ldots, R_{N+1}, \sigma_{q}^{2}\right\}$ & $2 N-M+1$ \\
\hline
\end{tabular}

In the case of $\sigma=0$, (17) turn into a formula of Wiener-Khintchine theorem.

$$
R_{k}=\int_{-\pi}^{\pi} \hat{S}_{q}(\omega) e^{j \omega k} d \omega,|k|=0, \ldots, M
$$

From this, the following is composed.

$$
\hat{S}_{q}(\omega)=\sum_{k=-\infty}^{\infty} R_{k} e^{-j \omega k}
$$

And, $\hat{S}_{q}(\omega)$ in (35) is defined as follows.

$$
\hat{S}_{q}(\omega)=\left.\frac{\sigma_{q}^{2}}{\sum_{n=0}^{N} b_{n} z^{-n} \sum_{s=0}^{N} b_{s}^{*} z^{s}}\right|_{z=e^{j \omega}}
$$

From (35), (36), the following composed.

$$
\frac{\sigma_{q}^{2}}{\sum_{n=0}^{N} b_{n} z^{-n} \sum_{s=0}^{N} b_{s}^{*} z^{s}}=\sum_{k=-\infty}^{\infty} R_{k} z^{-k}
$$

where $z=e^{j \omega}$. Here, in the right-hand side of an upper equation, if $k=r-n$, the following is composed.

$$
\begin{gathered}
\frac{\sigma_{q}^{2}}{\sum_{s=0}^{N} b_{s}^{*} z^{s}}=\sum_{n=0}^{N} b_{n} z^{-n} \sum_{r=-\infty}^{\infty} R_{r-n} z^{-(r-n)} \\
\sigma_{q}^{2} \sum_{s=0}^{\infty} h_{s} z^{s}=\sum_{r=-\infty}^{\infty}\left[\sum_{n=0}^{N} R_{r-n} b_{n}\right] z^{-r}
\end{gathered}
$$

where $h_{s}$ is impulse response. From the upper equation is considered the polynomial of $z$, the following is composed.

$$
\sum_{n=0}^{N} R_{r-n} b_{n}=\left\{\begin{array}{cc}
\sigma_{q}^{2} & r=0 \\
0 & 1 \leq r \leq N
\end{array}\right.
$$

Consequently, the following Yule-Walker equation is derived from $n$ is changed into $k$.

$$
-\sum_{k=0}^{N} R_{k-n} b_{k}+\sigma_{q}^{2} \delta_{r}=0, r=0, \ldots, N
$$

In addition, when $r^{\prime}=r+1$ and $r^{\prime}$ is changed into $r,(14)$ is derived as follows.

$$
\begin{array}{r}
f_{r}(\underline{y})=-\sum_{k=0}^{N} R_{r-k-1} b_{k}+\sigma_{q}^{2} \delta_{r-1}, \\
r=1, \ldots, N+1
\end{array}
$$


In (27), (15) is derived as follows from $r^{\prime}=$ $r+N-M$ and $r^{\prime} \rightarrow r$.

$$
\begin{aligned}
f_{r}(\underline{y})=\sum_{k=0}^{2 N-M+1-r} b_{k} b_{k+r+M-N-1} \\
-\frac{\sigma_{q}^{2}}{\sigma_{p}^{2}} \sum_{k=0}^{2 N-M+1-r} a_{k} a_{k+r+M-N-1}, \\
\quad r=N+2, \ldots, 2 N-M+1(41)
\end{aligned}
$$

Hence, the consistency of C-MCEM and CMCEMU is derived .

\section{Simulation}

We discuss the accuracy of C-MCEMU in comparison with C-MCEM with the constraints of known autocorrelations and Burg method. We estimate a spectrum to 8th order AR model.

First, we generate time series $x_{t}$ from 8 th order AR. model which has the following parameters.

Table 2: Parameters

\begin{tabular}{|c|c|c|c|}
\hline$b_{1}$ & $b_{2}$ & $b_{3}$ & $b_{4}$ \\
\hline 0.07 & 0.0692 & -0.0215 & -0.1171 \\
\hline$b_{5}$ & $b_{6}$ & $b_{7}$ & $b_{8}$ \\
\hline-0.245 & 0.6441 & 0.1308 & -0.0014 \\
\hline
\end{tabular}

$x_{t}$ is divided into two frames, the first frame's data is prior information, and we estimate the spectrum of the second frame's data. Where prior AR model's order $N=8$, the number of known autocorrelations $M=$ 6, sampling frequency $F s=22050[\mathrm{~Hz}]$, the number of sample per frame $l=256$. When we estimate the spectrum of second frame by C-MCEMU, let the spectrum of estimating by Yule-Walker estimating method be prior spectrum. Fig. 1-(a) shows true spectrum and prior spectrum. Fig. 1-(b) shows true spectrum, posterior spectrum for C-MCEM (MCE0) and C-MCEMU (MCE1). Fig. 1(c) is enlargement figure of around the first peak. Table. 3 shows the peak of each spectrum.

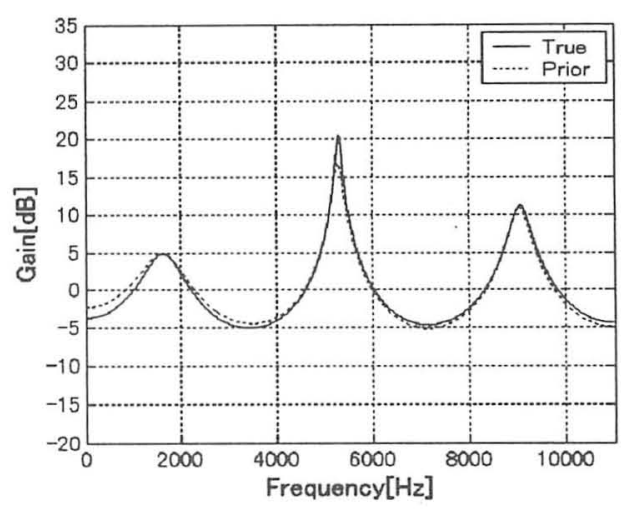

(a) True posterior spectrum and Prior spectrum

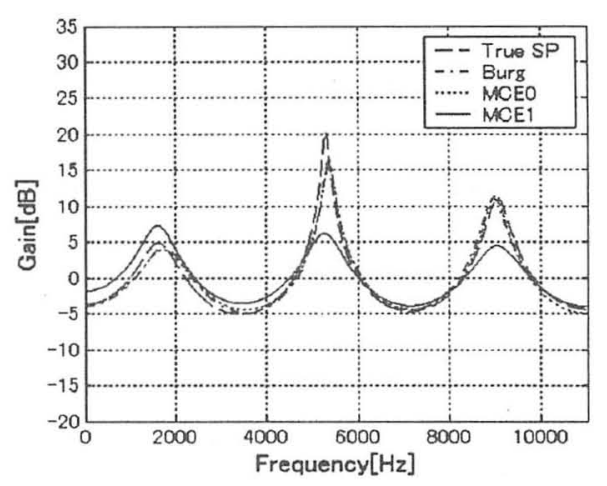

(b) Whole figure

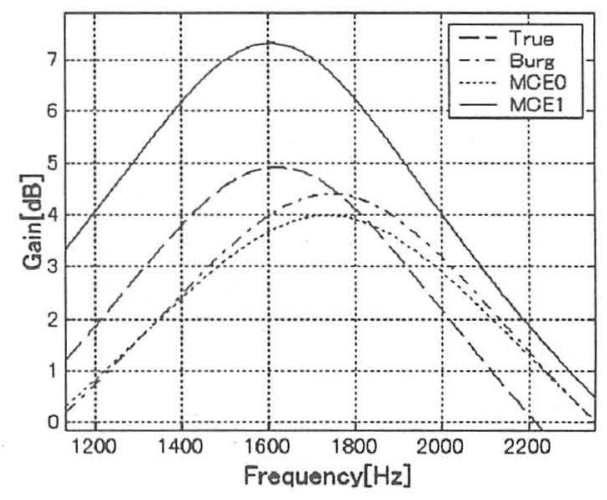

(c) Enlargement figure of around the first peak frequency

Fig. 1: Spectrum

Table 3: The peak frequency

\begin{tabular}{|l|c|c|c|c|c|}
\hline & True & Burg & MCE0 & MCE1 & Prior \\
\hline Peak1 [Hz] & 1621 & 1742 & 1731 & 1610 & 1610 \\
\hline Peak2 [Hz] & 5303 & 5358 & 5369 & 5259 & 5281 \\
\hline Peak3 [Hz] & 9063 & 8974 & 9007 & 9052 & 9041 \\
\hline
\end{tabular}


From Table. 3, we can find that the estimated spectrum by the C-MCEMU do not. have bias compared with others. However, estimation by C-MCEMU depends on the accuracy of estimating prior spectrum, and the number of the constraints $M$.

\section{Conclusion}

In this paper, we have developed the algorithm of the C-MCEM with uncertain autocorrelations (C-MCEMU) and derived consistency of C-MCEM and C-MCEMU. In comparison with C-MCEMU, C-MCEM and Burg method, we found that the estimated spectrum by the C-MCEMU do not have bias. However, accuracy of spectral estimation by C-MCEMU method depends on prior spectral estimation, and the number of the constraints $M$.

We will simulate by C-MCEMU to real speech data and apply a solution method replaced with the Newton's method which is a solution method of nonlinear simultaneous equations.

\section{References}

[1] K. Nakamuro, K. Tanaka, Y. Togawa, and S. Sugimoto. The Spectral Estimation of The Speech Applying Minimum Cross Entropy Method. Proc. of the 4\%th Annual Conference of the ISCIE, pp. 449450, (2003).

[2] J. E. Shore. Minimum Cross - Entropy Spectral Analysis. IEEE Trans. Acoust., Speech, Signal Processing, Vol. ASSP-29, pp. 230-237, (1981).

[3] R. W. Johnson and J. E. Shore. Minimum Cross - Entropy Spectral Analysis of Multiple Signals. IEEE Trans. Acoust., Speech, Signal Processing, Vol. ASSP-31, ก̃o. 3, pp. 574-582, (1983).

[4] A. Papoulis. Maximum Entropy and Spectral Estimation: A Review. IEEE
Trans. Acoust., Speech, Signal Processing, Vol. ASSP-29, No. 6, pp. 1176-1186.

[5] S. Sugimoto, T. Wada, and E. Nakatomi. Minimum Cross Entropy and Informational Approaches for a Spectrum Estimation. Proc. of "th, IFAC/IFORS Symp. on Identification and System Parameter Estimation, pp. 1727-1732, (1985).

[6] T. Wada, K. Nakamuro, and S. Sugimoto. A Spectral Estimation Algorithm Based on Minimum Cross Entropy Method. Proc. of SICE, pp. 262-267, (June 2002).

[7] S. Sugimoto and T. Wada. Spectral Expression of Information Measures of Gaussian Time Series and Their Relation to AIC and CAT. IEEE Trans. of Information Theory, Vol. IT-34, No. 4, pp. 625-631, (1988). 